\title{
A Década da Ciência Oceânica como Oportunidade de Justiça Azul no Sul Global
}

\author{
The Ocean Decade as an Opportunity to Promote Blue Justice in the Global South
}

DOI: https://doi.org/10.22456/2178-8839.113905

Ana Flávia Barros Platiau

Universidade de Brasília, Brasília, Brasil

anaflaviaplatiau@gmail.com

Leandra Regina Gonçalves

Universidade de São Paulo/Instituto Oceanográfico, São Paulo, Brasil goncalvesleandra@gmail.com

() (10)

Carina Costa De Oliveira

Universidade de Brasília, Brasília, Brasil

carinaoliveira@unb.br

\begin{abstract}
Resumo
As atividades das mulheres são geralmente subestimadas em áreas tradicionalmente dominadas por homens, e no oceano não é dife rente. $\mathrm{O}$ oceano tem sido visto como um ambiente masculino, principalmente na navegação, na pesca e nas ciências exatas. Como a aceleração da economia azul engendra riscos socioeconômicos para a humanidade, notadamente para as pessoas no Sul Global, e mais especificamente para as mulheres, defendemos as mudanças transformadoras debatidas no âmbito multilateral. Neste cenário, como a Década da ONU para a Ciência Oceânica pode contribuir para transformar barreiras à igualdade de gênero em oportunidades para a justiça azul? Nosso principal objetivo é destacar que a Década é baseada na ciência e no conhecimento, e pode ter papel central na promoção de uma economia azul mais sustentável, nos moldes da Agenda 2030. Por focar na "ciência que precisamos para o oceano que queremos”, ela incentiva a pesquisa participativa e interdisciplinar. Neste artigo de perspectiva, a abordagem é interdisciplinar para fomentar reflexão sobre o caminho para a justiça azul
\end{abstract}

Palavras-chave: Mulheres; Ciência Oceânica; Justiça Azul;

\begin{abstract}
The activities of women are usually underestimated in areas traditionally dominated by men. Thus, the ocean is not different. Ocean has been se en as a male environment, especially in navigation, fisheries and hard sciences. Because the acceleration of the blue economy brings socio ecological risks to humanity, notably to people in the so-called Global South, and particularly to women, we advocate for transformative change. How the UN Decade for Ocean Science may contribute to transform barriers to gender equity in opportunities to promote blue justice? Our main goal is to show that the Ocean Decade may play a role in fostering a more sustainable blue economy, in accordance with the UN 2030 Agenda, because it focuse s on "the science we need for the ocean we want", therefore encouraging participative and interdisciplinary research. Moreover, scientific research offers space for women to harness their preferences and priorities. In this perspective article, we employ an interdisciplinary approach to draw insights over the transformation pathway to blue justice.
\end{abstract}

Keywords: Women; Ocean Science; Blue Justice; 


\section{Introdução}

O oceano tem importância indiscutível para o Planeta, por sua contribuição à segurança alimentar, por prover diferentes serviços ecossistêmicos e por ser fonte de renda para diferentes comunidades costeiras (FAO, 2020). Na literatura recente, convencionou-se denominar “azul” a economia e a justiça concernentes aos oceanos. À medida que o interesse pelos recursos pesqueiros, pela mineração e pela biodiversidade aumenta, cresce também a competição entre diferentes atores econômicos, engendrando riscos de exclusão e desigualdade social. No Sul Global, o oceano tem relevância ainda maior para países de renda média ou baixa, dependendo do perfil de suas respectivas economias. Em 2013, estimou-se que cerca de um bilhão de pessoas de países em desenvolvimento dependiam de pescados como sua fonte primária de proteínas (Banco Mundial, 2015). Além disso, de acordo com os dados do Banco Mundial (2021), os países que apresentam maior disparidade econômica são os que também apresentam maiores desigualdades de gênero.

Para a construção de uma economia azul que incorpore as dimensões de justiça azul e equidade, Bennett et al (2021) elencaram dez desafios. Todos eles são importantes e conectados, a seguir:1) ocupação do oceano (dispossession and ocean grabbing); 2) justiça ambiental no contexto da poluição e do lançamento de resíduos; 3 ) degradação ambiental e redução dos serviços ecossistêmicos; 4) impactos no meio de vida dos pescadores de pequena escala; 5) perda do acesso aos recursos necessários para a segurança alimentar e o bem-estar; 6) distribuição desigual de benefícios econômicos; 7) impactos sociais e culturais; 8) marginalização das mulheres;9) violação dos direitos humanos e indígenas; e, 10) exclusão da governança. Assim, fica claro que o empoderamento das mulheres é condição sine qua non para a responder a estes desafios.

Neste contexto, como a Década das Nações Unidas para a Ciência Oceânica pode contribuir para transformar as barreiras à igualdade de gênero em oportunidades para promover a justiça e a igualdade, especialmente na pesquisa científica? Nossa hipótese é que com o início da Década do Oceano, consolidou-se a oportunidade de discutir como avançar para o desenvolvimento nos moldes da Agenda 2030 da Organização das Nações Unidas (ONU), com seus 17 Objetivos de DesenvolvimentoSustentável(ODS), no sentido de Kakar et al (2021) e mais especificamente Gjerde e Vierros (2021). Isto implica a oportunidade de tornar a economia azul mais equitativa, a fim de fortalecer a gestão sustentável dos recursos marinhos e a justiça azul (ODS 1, 2, 3, 6, 8, 10 e principalmente o14, relativo à vida na água), incluindo a igualdade de gênero (ODS 5).

A inclusão da igualdade de gênero na governança do oceano é um dos grandes desafios da Década da Ciência Oceânica, resultado de longo trabalho de diversas agências da ONU para o empoderamento de mulheres e meninas, como o Fundo de Desenvolvimento das Nações Unidas para a Mulher (UNIFEM), Programa das das Nações Unidas para para o Desenvolvimento (PNUD) e a Organização das Nações Unidas para a Educação, a Ciência e a Cultura (UNESCO), entre outras. Entretanto, a maior participação das mulheres nas atividades relacionadas ao oceano é recente, tendo sido incentivada no âmbito da Agenda 2030 da ONU, por meio de objetivos de desenvolvimento sustentável interrelacionados (LE BLANC, 2015). Sendo assim, se o ODS 5 busca alcançar a igualdade de gênero, ele pode ser implementado em consonância com o ODS 14, que trata do uso sustentável dos recursos marinhos com base na ciência, com iniciativas voltadas ao envolvimento de mulheres em vários aspectos da economia azul (LE BLANC et al., 2017). Entre eles, a meta 14.4 que indica planos de manejo da pesca baseados na ciência e a 14.7, que pretende aumentar os benefícios dos países menos desenvolvidos e as pequenas ilhas (small island developingstates, SIDS) por meio da gestão sustentável da pesca, aquicultura e turismo.

$\mathrm{O}$ artigo destaca as interfaces desse debate no que concerne diretamenteàs mulheres. Primeiro, o texto trata da economia e da justiça azul, conceitos relativamente recentes que destacam as desigualdades atuais entre o Norte e o Sul, ilustrando os desafios mencionados acima. Em seguida, discute o conceito de Sul Global, espaço no qual a desigualdade de gênero pode ser agravada se a economia azul continuar a crescer sem a justiça azul. Finalmentejoga luz sobre a Década da Ciência Oceânica para o Desenvolvimento Sustentável (2021-2030) da Organização das Nações Unidas (Década do 
Oceano) como uma oportunidade ímpar para que as mulheres registrem seus conhecimentos (científicos e tradicionais), prioridades e visões de mundo, com foco especial para o Brasil. O método é eclético, na linha de Lake (2013), focado em problemas da vida real, e não nos grandes debates teóricos das relações internacionais. Converge com a agenda de pesquisa sobre feminismo descrita por Guimarães (2021). O artigo traz abordagem interdisciplin ar entre as relações internacionais e o direito internacional público na grande área da ciência oceânica (ainda pouco estruturada no Sul Global), para analisar a literatura disponível sobre o tema, com fontes primárias e secundárias. Desta forma, o texto objetiva demonstrar a relevância da Década da Ciência Oceânica para se discutir a necessidade de uma economia azul mais justa e equitativa, tendo a equidade de gênero comoum de seus pilares.

\section{A Economia Azul e a Justiça Azul}

Embora o termo “economia azul” venha sendo usado de maneiras diferentes, entende-se aqui que é constituído de uma variada gama de setores econômicos e políticas relacionadas ao oceano, que juntos determinam como deve ser realizado o uso dos recursos oceânicos. A economia azul deveimplicar, no entanto, o gerenciamento da variável não apenas econômica, mas também social e ambiental (SCHUTTER et al., 2021; ANDRADE et al., 2020; BEIRÃO et al., 2020). Sendo assim, o conceito de economia azul deve promover o crescimento econômico, a inclusão social e a melhoria dos meios de subsistência e, ao mesmo tempo, garantir a proteção da vida marinha. Portanto, para que exista uma economia azul justa, a sustentabilidade deve ser central no ciclo das políticas públicas. Isso exige cuidadosa atenção a todas as decisões e suas implicações intersetoriais. Para essa integração, é necessário garantir que as políticas estejam alinhadas e sejam complementares (BENNET et al., 2021). É relevante indicar como o conceito tem sido verificado na prática, de que modo ele pode se conectar à justiça azul e como o tema tem sido abordado no Brasil.

Enquanto essa discussão conceitual sobre economia azul deslancha em fóruns internacionais e nacionais, na prática nota-se um aumento do interesse comercial pelos recursos marinhos, vivos e não vivos (FAO, 2020) e de forma acelerada (BLASIAK, 2020; JOUFFRAY et al., 2020), levando ao conceito de “aceleração azul”. Este é útil para destacar o escopo, a intensidade e a diversidade sem precedentes da exploração de recursos marinhos (JOUFFRAY et al., 2020). De fato, tal interesse não é recente e nem apenas comercial. Desde as primeiras civilizações, há registros de guerras de conquista e da valorização geopolítica, econômica, comercial e cultural do oceano, entre outras. Entretanto, o uso de novas tecnologias, como a localização de cardumes e cetáceos no alto mar por satélites, exige a análise cuidadosa de como a economia azul vem evoluindo no séculoXXI.

A economia azul possui características que podem aproximá-la ou afastá-la da justiça azul. Na segunda perspectiva, pode ser citado o acesso ao conhecimento científico oceânico. Embora tenha se desenvolvido no contexto da atual economia de dados (data driven economy), o conhecimento científico sobre o oceano e os seus recursos, bem como os serviços ecossistêmicos prestados e as interações das dinâmicas planetárias ainda são pouco conhecidos (MILLER, 2018). O fato é que os avanços tecnológicos permitem acesso a recursos que antes eram considerados inviáveis do ponto de vista da exploração econômica. Neste sentido, paradoxalmente, o acesso a recursos além da jurisdição nacional tem sido acelerado, ao passo que a pesquisa científica avança em ritmo muito mais lento, e tem permanecido concentrada em poucos países. Em consequência, estima-se que algumas espécies podem desaparecer mesmo antes de serem estudadas (WORM et al., 2006).

$\mathrm{Na}$ corrida para os recursos oceânicos, os países desenvolvidos tendem a usar suas vantagens institucionais, tecnológicas, científicas e comerciais para chegar primeiro aos recursos vivos e nãovivos. Obviamente, países como China, Coreia do Sul, Índia e Brasil também têm suas respectivas vantagens. Os dados apresentados por Jouffray et al (2020) sobre aceleração azul comprovam tal afirmativa, como a exploração de petróleo e gás offshore, a navegação, o turismo, os cabos submarinos, a dessalinização da água, etc. Por exemplo, entre aqueles que têm acordos de exploração com a Autoridade Internacional para os Fundos Marinhos (ISBA), o Brasil é o único latino-americano. 
O segundo aspecto que afasta a economia da justiça azul é o fato de que ela é dificilmente regulada. Os diversos tratados multilaterais sob a égide da ONU levaram a uma governança fragmentada (BARROS -PLATIAU; MALJEANDUBOIS, 2015), porém que teve um excelente ponto de partida com a adoção da Convenção das Nações Unidas sobre o Direito do Mar (CNUDM ou UNCLOS em inglês) há quase quarenta anos. Em outros termos, finda a ordem bipolar e reforçado o sistema ONU, a governança do oceano permaneceu profundamente fragmentada e lacunária. Atualmente, dois acordos multilaterais estão em vias de negociaçãona ONU: um sobre o Código de Mineração sob os auspícios da Autoridade Internacional dos Fundos Marinhos e outro sobre a Biodiversidade Marinha Além da Jurisdição Nacional (BBNJ da sigla em inglês). Ambos são importantes, porém apenas parcialmente conectados do ponto de vista legal e diplomático. Indubitavelmente, eles são resultado de conflitos de interesse ambientais e econômicos. Além disso, ambos são desprovidos de conhecimento científico robusto e conclusivo (LEVIN, 2020).

Ademais, há uma grande assimetria regional, sendo que países do Norte desenvolveram arcabouços normativos relativamente efetivos e recentes, como as Organizações regionais de ordenamento pesqueiro (OROPS), enquanto os demais têm resultados extremamente limitados na cooperação internacional, como no caso brasileiro (TOMÉ, 2020). Consequentemente, a pesca ilegal, não-declarada e não-regulamentada (INN) constitui um enorme desafio no Atlântico Sul e no Oceano Antártico.

Ainda nesse contexto de difícil regulação, cita-se a taxonomia dos atores interessados pelo tema (stakeholders). $\mathrm{O}$ setor privadoé essencial nos debates para a governança do oceano. Tanto no setor alimentar, energético e sanitário, como na pesquisa, na biotecnologia, as grandes empresas têm papel central (BLASIAK, 2020; JOUFFRAY et al., 2020). Logo, a participação ampla de atores do mercado nos processos decisórios precisa ser regulada de forma transparente e efetiva.

Outro fator que distancia a economia da justiça azul é o financiamento das atividades (PEREIRA, 2020), principalmente os subsídios à pesca, tratados na Meta 14.6 da Agenda 2030. Apesar de existirem iniciativas envolvendo atores públicos e privados, tais como Principles for Responsible Banking e The Sustainable Blue Economy Finance Principles, a sua operacionalização ainda é incipiente (JOUFFRAY et al., 2020). Para que a economia azul seja mais equitativa e sustentável, recursos e tecnologia não bastam. Estabilidade nacional e combate à corrupção também são essenciais (CISNEROS-MONTEMAYOR et al., 2021). Eventos internacionais têm promovido debates sobre todas estas questões, incentivados pela Década do Oceano.

A primeira Conferência sobre Economia Azul Sustentável foi realizada no Quênia em novembro de 2018, e reuniu centenas de especialistas em oceanos para discutir como utilizar os recursos oceânicos de forma sustentável.A conferência indicou alguns temas -chaves para serem trabalhados, que podem promover a conexão entre a economia e a justiça azul: 1 . reforçar a interface ciência-política para as atividades relacionadas à economia azul visando aprimorar a tomada de decisão; 2. fortalecer os mecanismos de governança para uma economia azul sustentável, garantindo a participação das partes interessadas na política e na tomada de decisões; 3 . promover atividades econômicas consistentes com conservação e uso sustentável à longo prazo do oceano, mares, lagos e rios; 4. fortalecer a responsabilidade corporativa social e ambiental; 5. aprimorar o conhecimento e compreensão sobre ovalor do mercado para bens e serviços não mercantis da economia azul; 6. realinhar o financiamento para apoiar atividades econômicas inclusivas que melhorem a saúde da economia azul; 7. criar mercados para indústrias emergentes e inovadoras relacionadas à economia azul. Nota -se que as preocupações ainda estão bem centradas no desenvolvimento econômico, e menos em sua interdependência com as dimensões sociais e ambientais. Entendemos que o papel das mulheres nestas iniciativas precisa ser devidamente destacado.

Outra iniciativa global que vale mencionar é o Painel de Alto Nível para uma Economia do Oceano Sustentável (em inglês Ocean Panel). Trata-se de uma iniciativa de 14 líderes mundiais que estão construindo um impulso para uma economia oceânica sustentável, objetivando construir um futuro melhor e mais resiliente para as pessoas e o planeta. Estabelecido em setembro de 2018, o Ocean Panel tem trabalhado com governos, empresas, instituições financeiras, a 
comunidade científica e a sociedade civil para catalisar e dimensionar soluções ousadas e pragmáticas em política, governança, tecnologia e finanças para, em última análise, desenvolver uma agenda de ação para a transição para uma economia oceânica sustentável. O painel é co-presidido pela Noruega e Palau, e composto por membros da Austrália, Canadá, Chile, Fiji, Gana, Indonésia,Jamaica, Japão, Quênia, México, Namíbia, Noruega, Palau e Portugal, sendo apoiado pela ONU. Nota-se ainda que embora a discussão sobre economia azul venha ganhando força no mundo, inclusive em níveis mais altos da tomada de decisão na primeira conferência sobre o tema, o Brasil compareceu, porém não se comprometeu com metase atividades.

No caso do Brasil, pode-se destacar o pleito da extensão da plataforma continental e o conceito de Amazônia Azul como indicadores do interesse nacional pelos recursos biológicos e minerais marinhos (MARRONI, 2013; PEREIRA, 2021; WEBSTER et al.,2020). Um estudo recente afirma que a "Economia do Mar" no Brasil gera um valor adicionado anual de mais de 1,18 trilhão de Reais, gerando 19 milhões de empregos (CARVALHO, 2018), e essa economia corresponde a 19\% do PIB brasileiro.

Em evento promovido pelo Ministério de Ciência e Tecnologia e Inovação - 1o Fórum Brasileiro de Ciência, Tecnologia e Inovação para os Oceanos, o termo "economia azul" esteve presente em diversas falas, em sua maioria, se referindo à gigante lacuna de conhecimento e arcabouço regulatório sobre o tema no Brasil. No entanto, esse debate ainda é incipiente e pode se beneficiar de um alargamento do conceito e uma ampla discussão com demais setores da sociedade. Nesse sentido, faz-se necessário aprofundar o tema da economia azul não só para o Brasil, mas para todo o Sul Global, com foco na sustentabilidade, aproveitando também o momentum que vem sendo fomentado pela Organização das Nações Unidas. O contexto brasileiro está espelhado no Sul Global, tema esse que seráobjeto de análise abaixo.

\section{A justiça azul no Sul Global e a inclusão da igualdade de gênero}

A apropriação acelerada de recursos marinhos tem fortalecido a arquitetura global que engendra novas desigualdades e desafios (BENNETT et al., 2021). Entretanto, a comunidade científica ainda não conseguiu estimar de forma adequada ovalor de tais recursos, e como tem sido a distribuição entre eles em escala planetária (JOUFFRAY et al., 2020). Por isso, a justiça é central no desenvolvimento de uma economia azul mais sustentável. Riscos globais, como por exemplo no caso das mudanças climáticas, afetam de forma muito desigual os países que mais dependem de recursos do oceano, afetando diretamente o Sul Global. As potências tecnológicas aumentam os riscos para o Planeta, seja com testes nucleares, a decisão japonesa de despejar água radioativa do acidente de Fukushima no oceano em 2021, a pesca industrial subsidiada, ou a mineração no Ártico, entre tantas atividades reprocháveis. Diante desse contexto, é relevante analisar as características do Sul Global no contexto da conexão entre justiça e economia azul e os desafios da inclusão da igualdade de gênero.

\section{O Sul Global}

O Sul Global geralmente tem três características em comum. Primeiramente, uma parteimportante da economia é informal, com oportunidades para abusos de direitos humanos e trabalhistas, além de corrupção e violências. Segundo, na maioria das vezes são paíse s com sociedades desiguais, nas quais os homens sustentam a casa e as mulheres têm salários menores, ou seja, a desigualdade de gênero é geralmente grave. Ademais, em terceiro, a fragilidade institucional não permite a proteção adequada das pessoas mais vulneráveis. Se uma indústria causar danos para a atividade de uma comunidade local, como as marisqueiras, por exemplo, dificilmente poderão recorrer à justiça e ter chances de ganhar a

\footnotetext{
No presente estudo, a economia do mar será chamada de "economia azul” para estar mais alinhada com a definição da Organização das Nações Unidas sobre "blue economy", e que traz componentes em sua definição e análise voltadas à promoção do desenvolvimento racional e sustentável.
} 
causa, em razão da desconexão de poder entre aqueles que sofrem o impacto e aqueles que tomam as decisões (GONÇALVES etal., 2020).

O Sul Global faz sentido na pesquisa sobre a justiça azul não apenas pelo impacto que essa agenda emergente pode ter, mas também porque a forma pela qual a agenda é construída pode incluir as epistemologias do Sul e ser mais plural. Logo, a perspectiva do Sul é central para o debate sobre economia e justiça azuis, garantindo a inclusão de temas centrais, como a equidade de gênero.

No entanto, apesar de sua longa trajetória, o conceito de "Sul Global” ainda nãoé ponto pacífico (TENNISWOOD, 2018). Nas agendas de pesquisa de relações internacionais e no direito internacional público, o termo aparece com frequência, mas suscita reações adversas. Na década de 1960, com os processos de descolonização e a Guerra Fria, houve a separação entre os países ditos “desenvolvidos” (aqueles diretamente envolvidos na ordem bipolar) e os demais (o Terceiro Mundo). As lideranças políticas e intelectuais, como a Cepalina, contribuíram para a análise deste fenômeno, empregando conceitos como centro e periferia, a fim de mostrar como os termos de trocas internacionais eram desfavoráveis aos países do Sul, na época também chamados de "subdesenvolvidos” nojargão da ONU.

Diversos fatores contribuíram para o debate que culminou com a Resolução 3.201, de $1^{\circ}$ de Maio de 1974, da Assembleia Geral da ONU (AGNU), em 1974, concernente à criação da Nova Ordem Econômica Internacional (NOEI), bem como o seu Plano de Ação. Apesar da Resolução não ter tido o efeito esperado, ela serviu de base para a aproximação de países do Sul à época, e novas iniciativas foram propostas pela maioria dos membros na AGNU, ou seja, os países em desenvolvimento, situados no Hemisfério Sul.

Certo é que o conceito de Sul Global é muito mais complexo do que a história recente do multilateralismo Onusiano. Ele corresponde a uma longa história de divisão do mundo entre have e have nots, com uma narrativa de que mapa do mundo geralmente pode ser dividido em dois grandes grupos: os colonizadores e os colonizados; os que comandam e os que obedecem, os que decidem e os que são ignorados em diversos processos decisórios. Entretanto, os países do Sul Global não são apenas vítimas de países mais poderosos. São também vítimas dos seus próprios sistemas e mazelas, com suas respectivas fragilidades institucionais.

Evidentemente, o Sul Global não corresponde a um mapa estático e simples. Ao contrário, o grupo de países varia em função dos períodos históricos, narrativas políticas e dados analisados. Portanto, não há recor te que permita uma imagem clara e precisa do que seria o Sul Global. Sua constituição depende do recorte escolhido, da abordagem empregada e do recorte temático.

Este é apenas um dos argumentos dos detratores do conceito de Sul Global, a sua existência real. Alguns deles preferem usar os grupos efetivamente constituídos, como o G77/China, ou até mesmo o Terceiro Mundo, quando existia. Outro argumento crítico é baseado na assimetria dentro do suposto Grupo, uma vez que os países emergentes, e mais especificamente a China, não deveriam mais ser considerados como países do Sul. Terceiro, se o critério escolhido for a prioridade para ações coletivas a partir das pessoas em situação de extrema pobreza, China e Índia combinadas historicamente concentraram a maior população em situação de extrema pobreza. Contudo, os dois países são também potências tecnológicas para pesquisas oceânicas, polares, nucleares e espaciais. Finalmente, se "Sul Global” for um conceito meramente político e politizado, ele ainda permanece extremamente frágil. Entretanto, alguns países do Sul conseguiram superar os desafios do desenvolvimento nacional e aumentaram de forma extraordinária o seu índice de desenvolvimento humano (IDH/PNUD). Entre eles, a Coréia do Sul se destaca, e mais recentem ente a China.

Nesta perspectiva, a Década do Oceano deve promover uma economia azul justa e igualitária que: (1) discuta os desafios relevantes de curto e longo prazo causados pela aceleração azul (2) dialogue com diferentes setores soluções para um futuro próximo, (3) inclua e compreenda o papel de todos os atores na ciências do mar e (4) compreenda o papel dos países do Sul Global no desenho e na arquitetura institucional da economia azul e (5) promova a equidade de gênero e uma 
maior presença das mulheres nos setores afins do mar (e.g: navegação, turismo, pesca, ciência e entre outros). A seguir discutimos o exemplomais específico das mulheres.

\section{A justiça azul por meio da igualdade de gênero na pesquisa científica}

Foi discutido como a aceleração azul tem trazido desafios para o Sul Global no desenho de instituições para uma efetiva governança do oceano, que possa trazer mais justiça azul e igualdade. Como solução, Cisneros-Montemayor et al (2021) sugerem a cooperação internacional transcalar e investimentos focados (targeted investments and cross-scale cooperation). As respostas para os desafios citados passam necessariamente pelo conhecimento científico e tradicional, e acessível. Neste contexto de grandes mudanças no cenário global, cabe discutir o lugar das mulheres na ciência oceânica, contrastado também com representantes doSul Global como o Brasil.

O Global Ocean Science Report revelou que em 2013, estimava-se que as mulheres correspondiam a 38\% do total de pesquisadores, enquanto o total para pesquisas em geral era em torno de $28 \%$. Entretanto, há uma grande variação entre países, entre os $4 \%$ na Mauritânia e os $62 \%$ na Croácia. Outra grande variação refere -se às áreas da pesquisa. Cerca de 75\% dos pesquisadores envolvidos com tecnologia e observação oceânica são homens (KIRSTEN et al., 2018).

Mundialmente, as mulheres enfrentam barreiras profissionais, tais como financiamento, contratação, licença maternidade e até a promoção a cargos seniores (GROGAN, 2019). Ainda como barreira, em média, os homens publicam mais artigos do que mulheres (GIAKOUMI et al., 2021). As mulheres têm menos sucesso do que os homens ao se candidatar a financiamento público de pesquisa (VAN DER LEE; ELLEMERS, 2015), e taxa mais baixa com base em candidaturas de empregoidênticas apresentadas com um nome masculino (MOSS-RACUSIN et al., 2012). Giakoumi et al (2021) apresentam diferentes evidências de que o preconceito de gênero na ciência está profundamente enraizado e se auto perpetua pelos preconceitos estruturais.

No Brasil, apesar do número de mulheres com diplomas de curso superior ter aumentado significativamente, isso ainda não se reflete no mercado de trabalho e nem na pesquisa científica. Há áreas que ainda são predominantemente ocupadas por homens, principalmente nas engenharias (FAPESP, 2021). No País ainda não há dados disponíveis e sistematizados sobre mulheres pesquisadoras no campo das ciências do mar. Recentemente, foilançado oX Plano Setorial para Recursos do Mar (BRASIL, 2020), porém o tema não foi proposto em ações futuras e nem foram apresentados dados sobre a porcentagem de pesquisadoras mulheres. A igualdade de gênero não está na ordem das prioridades do planejamento, da coordenação e da condução das atividades dos diferentes atores que possuem legítimos interesses ligados ao mar, sob a égide da Comissão Interministerial para os Recursos do Mar. O último Relatório de Atividades de 2019 e o Plano de Trabalho e Orçamento 2020 do Grupo Técnico Formação de Recursos Humanos em Ciências do Mar (PPG-Mar) também não apresentou linhas de ação voltadas ao equilíbrio de gênero no Brasil (PPGMAR, 2019), fato que poderá ser alterado com a criação do GT-Humanidades.

Essa ausência de prioridade e de dados para servirem de base para planejamento de políticas públicas dificulta ações voltadas à implementação da Agenda 2030 e da construção de uma economia azul justa e inclusiva no Brasil. É relevante destacar que durante as reuniões preparatórias para a Década da Ciência Oceânica, realizadas pelo MCTI, em todas as cinco regiões, a maior parte dos participantes eram mulheres (BRASIL, 2021). Evidencia -se, portanto, uma forte desconexão entre a realidade e os projetos institucionais para o campo das ciências do mar.

A maior participação das mulheres pode contribuir com a justiça e a equidade na economia azul (GONÇALVES e CHAMORRO, 2021). Como soluções, o investimento global e local na economia azul deve priorizar e fomentar o conhecimento e o seu acesso, por meio de uma governança efetiva e apropriada, cujo foco seja melhorar a equidade social e reduzir os riscos de mudanças climáticas e de perda de biodiversidade (CISNEROS-MONTEMAYOR et al., 2021). Neste contexto, a inclusão de questões sociais, como a igualdade de gênero, é fundamental para que novos rumos sejam tomados com relação ao desenvolvimento de setores da economia azul, haja vista que há muita disparidade de competências e 
recursos entre os países (CISNEROS-MONTEMAYOR et al., 2021). Ainda persistem barreiras pessoais, culturais e institucionais para as mulheres, apesar de algumas iniciativas para promover a igualdade de gênero (KIRSTEN et al., 2018). Nesta perspectiva, a Década do Oceano deve promover uma economia azul justa e igualitária que: (1) discuta os desafios relevantes de curto e longo prazo causados pela aceleração azul (2) dialogue com diferentes setores e soluções para um futuro próximo, (3) inclua e compreenda o papel das mulheres na ciências do mar e (4) compreenda opapel dos países do Sul Global no desenho e na arquitetura institucional da economia azul.

\section{Conclusão}

No tema concernente ao oceano, tradicionalmente predominam homens e atividades masculinas, embora a participação feminina esteja aumentando na ciência, ainda de forma bastante desigual nos diversos países. Neste cenário, a economia azul traz riscos de exclusão para o Sul Global em geral, e para as mulheres, em particular. Logo, a Década do Oceano oferece uma arena multilateral para discutirmos como promover a justiça azul nos moldes da Agenda 2030 e de outras iniciativas relacionadas.

O oceano revela o paradoxo de ser uma fonte de riqueza e saúde em escala planetária, mas ainda há muitoo que avançar nos diferentes campos do conhecimento e de políticas públicas para o desenvolvimento sustentável. A economia azul, em acelerado crescimento nas últimas décadas, tem levantado interesses em diversos setores, notadamenteno setor de pesquisa, desenvolvimento e inovação. Em outros termos, a exploração comercial de recursos marinhos tem aumentado sem que a ciência tenha tido tempo de subsidiar os processos decisórios e regulatórios de forma adequada, começando pela pesca e mineração. Logo, a economia azul revela novas possibilidades para que as presentes e futuras gerações transformem barreiras em oportunidades. Entretanto, se a economia azul permanecer desatrelada d os imperativos éticos da justiça azul, poderá trazer mais riscos de exclusão, violência, degradação ambiental e concentração de renda.

Neste contexto, a Década do Oceano se apresenta como uma oportunidade de construir uma economia azul pautada em outras dimensões para que, ao invés de reproduzir injustiças ambientais e sociais, possa inovar e construir uma economia azul mais justa e inclusiva. Argumentou-se aqui que a economia azul precisa considerar, dentre outros aspectos, a igualdade de gênero, no sentido de integrar o ODS 5 e o ODS 14 da Agenda 2030. Ademais, faz-se igualmente necessário considerar o papel das mulheres nas ciências do oceano. A participação delas ainda é incipiente, mas está em crescimento, e poderá estimular novos modelos de desenvolvimento e soluções inovadoras. Neste contexto, o Brasil tem a oportunidade de promover mudanças nas políticas nacionais e fortalecer este debate no Sul Global. Baseados na ciência e no conhecimento, os processos decisórios podem ser mais integrativos entre setores e jurisdições.

\section{Referências}

ANDRADE, Israel; BARROS-PLATIAU, Ana Flávia; CÂMARA, Paulo Eduardo; HILLEBRAND, Giovanni. Economia do mar: desafios e possibilidades para o Brasil na Amazônia Azul. Revista da Escola Superior de Guerra. Rio de Janeiro, v. 35, p. 50 - 77, 2020.

BANCO MUNDIAL, Global Partnership for Oceans, 2015. Disponível em: Infographic: Our Living Oceans (worldbank.org). Acesso em 23 julho 2021

BANCO MUNDIAL. Women, Business and the Law 2021. Washington, DC: World, 2021. Doi:10.1596/978-1-4648-1652-9.

BEIRÃO, André Panno; MARQUES, Miguel; RUSCHEL, Rogério. (Orgs.) O Valor do Mar. Uma Visão Integrada dos Recursos do Oceano do Brasil. São Paulo: Essential Idea, $2^{\mathrm{a}}$ edição, 2020.

BENNETT, Nathan; BLYTHE, Jessica;WHITE, Carole; CAMPERO, Cecilia. Blue growth and blue justice: Ten risks and solutions for the ocean economy. Marine Policy, 125, 2021, 104387.

BRASIL. Aprova o X Plano Setorial para os Recursos do Mar. DECRETO No 10.544, DE 16 DE NOVEMBRO DE 2020. Disponível em: https://www.marinha.mil.br/secirm/xpsrm. Acesso em 21 maio 2021. 
BRASIL. Relatório das Oficinas regionais e preparatórias para a Década da Ciência Oceânica para o Desenvolvimento Sustentável. 2021. Disponível em: http://decada.ciencianomar.mctic.gov.br/documentos/. Acesso em 21 maio 2021.

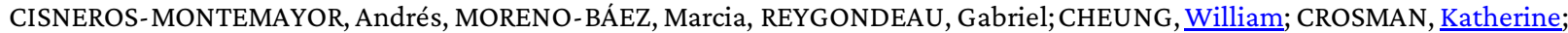

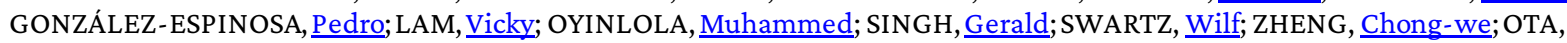
Yoshitak. Enabling conditions for an equitable and sustainable blue economy. Nature, 591, p. 396-401, 2021. doi.org/10.1038/s41586 021-03327-3

JOUFFRAY, Jean-Baptiste; BLASIAK, Robert; NORSTROM, Albert, ÖSTERBLOM, Henrik, NYSTROM, Magnus. The Blue Acceleration: The Trajectory of Human Expansion into the Ocean. One Earth, volume 2, issue 1, 2020, pages 43-54. Disponível em: https://doi.org/10.1016/j.oneear.2019.12.016. Acesso em 21 maio 2021.

FAPESP. Títulos de doutorado no Brasil. Seção dados. 2021. Disponível em: https://revistapesquisa.fapesp.br/titulos - de-doutoradono-brasil/. Acesso em 10 maio 2021.

GIAKOUMI, Sylvaine; PITA, Cristina; COLL Marta; FRASCHETTI, Simonetti; GISSI, Elena; KATARA, Isidora; LLORET - LLORET, Elena; ROSSI, Francesca; PORTMAN, Michelle; STELZENMÜLLER, Vanessa; MICHELI, Fiorenza. Persistent gender bias in marine science and conservation calls for action to achieve equity. Biological Conservation, 257, 2021, 109134.

GJERDE, Kristina; VIERROS, Marjo. Achieving SDG 14. Time for a Global Ocean Approach. In: KAKAR, Narinder; POPOVSKI, Vesselin; ROBINSON, Nicolas (Eds). Fulfilling the Sustainable Development Goals. Routledge, 2021.

GONÇALVES, Leandra; CHAMORRO, Paulina. Women and girls: Let's transform the ocean by including everyone (commentary). Mongabay, 2021. Disponível em: https://news.mongabay.com/2021/02/women-and-girls-lets-transform-the-ocean-by-includingeveryone-commentary/?utm_medium=Social\&utm_source=Twitter\#Echobox=1613067767.Acesso em 21 maio 2021 .

GOSR. Global Ocean Science Report, 2020.IOC, UNESCO. Disponível em: https://en.unesco.org/gosr. Acesso em 21 maio 2021.

GROGAN, Kathleen. How the entire scientific community can confront gender bias in the workplace. Nat. Ecol. Evol., 3, 2019 , p. 3.

GUIMARÃES, Feliciano. Teoria das Relações Internacionais. Editora Contexto, 2021.

KAKAR, Narinder; POPOVSKI, Vesselin; ROBINSON, Nicholas (Eds). Fulfilling the Sustainable Development Goals. Routledge, 2021.

LAKE, David. Theory is dead, long live theory: The end of the Great Debates and the rise of eclecticism in International Relations. European Journal of International Relations, 2013. Disponível em: https://doi.org/10.1177/1354066113494330. Acesso em 25 maio 2021.

LE BLANC, David. Towards Integration at Last? The Sustainable Development Goals as a Network of Targets. Sustain. Dev. 23, 2015, 176-187. Disponível em: https://doi.org/10.1002/sd.1582. Acesso em 21 maio 2021.

LE BLANC, David; FREIRE, Clovis; VIERRO Marjo. Mapping the linkages between oceans and other Sustainable Development Goals: A preliminary exploration. United Nations Dep. Econ. Soc. Aff. 1, 34, 2017.

LEVIN, Lisa;AMON, Diva; LILY, Hannah. Challenges to the sustainability of deep-seabed mining. Nature Sustainability 3, 784-794, 2020.Disponível em: https://doi.org/10.1038/s41893-020-0558-x. Acesso em 21 maio 2021.

MARRONI, Etiene. Política Internacional dos Oceanos. O caso Brasileiro sobre o processo diplomático para a plataforma continental estendida. Tese de doutorado (em Ciência Política). Universidade Federal do Rio Grande do Sul, Brasil, 2013, 361 p.

MILLER, Kathryn; THOMPSON, Kirsten; JOHNSON, Paul; SANTILLO, D. An Overview of Seabed Mining Including the Current State of Development, Environmental Impacts, and Knowledge Gaps. Frontiers in Marine Science, 4:418, p. 1-24, 2018. https://doi.org/10.3389/fmars.2017.00418.

MOSS-RACUSIN, Corinne; DOVIDIO,John; BRESCOLL, Victoria; GRAHAM, Mark; HANDELSMAN,Jo. Science faculty's subtle gender biases favor male students. Proceedings of the National Academy of Sciences, vol 109, 41, 2012, 16474-16479.

PEREIRA, Mariana. Economia Azul: O Caminho para Eficiência Econômica, Social e Ambiental das Atividades Produtivas Baseadas nos Oceanos. Dissertação (Mestrado em Economia), Universidade de Brasília, Brasil, 2020,149 p.

PEREIRA, Alexandre. Brazil advances over the Area: The inclusion of the Rio Grande Rise within the Brazilian outer continental shelf and its consequences for other states and for the common heritage of mankind. Marine Policy, 125, $2021,104399$.

PPGMAR. Relatório de Atividades 2019 Plano de Trabalho e Orçamento 2020 Brasília, março de 2020, 2019. Disponível em: https://cienciasdomarbrasil.furg.br/images/relatorios/Relatorio2019a2019ePlanejamento2020.pdf. Acesso em 25 de julho de 2021.

SCHUTTER, Marleen; HICKS, Christina; PHELPS,Jacob; WATERTON, Claire. The blue economy as a boundary object for hegemony across scales. Marine Policy, 132, 2021. Disponível em: https://doi.org/10.1016/i.marpol.2021.104673. Acesso em 25 de julho de 2021

TENNISWOOD, Sam. Power and Fragmentation in Global Governance Architectures: Global North vs. Global South at the Biodiversity Beyond National Jurisdiction Negotiations. Dissertação (Mestrado em Desenvolvimento Sustentável), Universidade de Utrecht, Holanda, 2018, 116 p. 
VAN DER LEE, Romy; ELLEMERS, Naomi. Gender contributes to personal research funding success in The Netherlands. Proceedings of the National Academy of Sciences, v. 112, n. 40, p. 12349-12353,2015.

WEBSTER, D. G.; GONÇALVES, Leandra; KIM, Rakhyun, BAILEY, Jennifer. How power disconnects may affect the outcome of the ongoing BBNJ negotiations? In: BARROS-PLATIAU, Ana Flávia; OLIVEIRA, Carina. Conservação dos recursos vivos em áreas além da jurisdição nacional : BBNJ e Antártica . Rio de Janeiro: Lumen Juris, 2020.

WORM, Boris; BARBIER, Edward; BEAUMONT, Nicole; DUFFY, J. Emmett; FOLKE, Carl; HALPERN, Benjamin; JAKSON,Jeremy; LOTZE, Heike; MICHELI, Fiorenza; PALUMBI, Stephen; SALA, Enric; SELKOE, Kimberley; STACHOWICZ,John; WATSON, Reg. Impacts of biodiversity loss on ocean ecosystem services. Science, v. 314, p. 787-790, 2006.

\begin{tabular}{|c|c|}
\hline Ana Flávia Barros Platiau: & $\begin{array}{l}\text { Conceituação; Metodologia; Validação; Curadoria de dados; Administração do projeto; Visualização; Análise formal; Recursos; } \\
\text { Obtenção de financiamento; Software; Investigação; Supervisão; Escrita (primeira redação); Escrita (revisão e edição); }\end{array}$ \\
\hline Leandra Regina Gonçalves: & $\begin{array}{l}\text { Conceituação; Metodologia; Validação; Curadoria de dados; Administração do projeto; Visualização; Análise formal; Recursos; } \\
\text { Obtençâo de financiamento; Software; Investigação; Supervisão; Escrita (primeira redação); Escrita (revisão e edição); }\end{array}$ \\
\hline Carina Costa De Oliveira: & Conceituação; Metodologia; Análise formal; Investigação; Escrita (revisão e edição); \\
\hline
\end{tabular}

